\title{
Statistical Summability through de la Vallée-Poussin Mean in Probabilistic Normed Spaces
}

\begin{abstract}
Ayhan Esi
Department of Mathematics, Science and Art Faculty, Adiyaman University, 02040 Adiyaman, Turkey

Correspondence should be addressed to Ayhan Esi; aesi23@hotmail.com

Received 13 February 2014; Revised 31 March 2014; Accepted 1 April 2014; Published 16 April 2014

Academic Editor: Biren N. Mandal

Copyright (C) 2014 Ayhan Esi. This is an open access article distributed under the Creative Commons Attribution License, which permits unrestricted use, distribution, and reproduction in any medium, provided the original work is properly cited.

Two concepts-one of statistical convergence and the other of de la Vallée-Poussin mean-play an important role in recent research on summability theory. In this work we define a new type of summability methods and statistical completeness involving the ideas of de la Vallée-Poussin mean and statistical convergence in the framework of probabilistic normed spaces.
\end{abstract}

\section{Introduction, Definitions, and Preliminaries}

Fast [1] presented the following definition of statistical convergence for sequences of real numbers. Let $K \subseteq \mathbb{N}$, the set of natural numbers, and $K_{n}=\{k \leq n: k \in K\}$. The natural density of $K$ is defined by $\delta(K)=\lim _{n} n^{-1}\left|K_{n}\right|$ if the limit exists, where $\left|K_{n}\right|$ denotes the cardinality of $K_{n}$.

The sequence $x=\left(x_{j}\right)$ is said to be statistically convergent to the number $\ell$ if for every $\epsilon>0$ the set $K_{\varepsilon}:=\{k \in \mathbb{N}$ : $\left.\left|x_{k}-\ell\right| \geq \varepsilon\right\}$ has natural density zero; that is, for each $\epsilon>0$,

$$
\lim _{n} \frac{1}{n}\left|\left\{j \leq n:\left|x_{j}-\ell\right| \geq \epsilon\right\}\right|=0
$$

Note that every convergent sequence is statistically convergent to the same limit, but its converse need not be true.

In 1985, Fridy [2] has defined the notion of statistically Cauchy sequence and proved that it is equivalent to statistical convergence and since then a large amount of work has appeared. Various extensions, generalizations, variants, and applications have been given by several authors so far, for example, [3-8] and references therein. In the recent past, Mursaleen [9] presented a generalization of statistical convergence by using de la Vallée-Poussin mean which is known $\lambda$-statistical convergence and further studied by Çolak and Bektas $[10,11]$. For more details related to this concept we refer to $[12-18]$.
Let $\lambda=\left(\lambda_{n}\right)$ be a nondecreasing sequence of positive numbers tending to $\infty$ such that

$$
\lambda_{n+1} \leq \lambda_{n}+1, \quad \lambda_{1}=0 .
$$

The generalized de la Vallée-Poussin mean is defined by

$$
t_{n}(x)=: \frac{1}{\lambda_{n}} \sum_{j \in I_{n}} x_{j}
$$

where $I_{n}=\left[n-\lambda_{n}+1, n\right]$.

A sequence $x=\left(x_{j}\right)$ is said to be $(V, \lambda)$-summable to a number $\ell$ if

$$
t_{n}(x) \longrightarrow \ell \text { as } n \longrightarrow \infty \text {. }
$$

In this case $\ell$ is called $\lambda$-limit of $x$.

Let $K \subseteq \mathbb{N}$ be a set of positive integers; then

$$
\delta_{\lambda}(K)=\lim _{n} \frac{1}{\lambda_{n}}\left|\left\{n-\lambda_{n}+1 \leq j \leq n: j \in K\right\}\right|
$$

is said to be $\lambda$-density of $K$.

In case $\lambda_{n}=n, \lambda$-density reduces to the natural density. Also, since $\left(\lambda_{n} / n\right) \leq 1, \delta(K) \leq \delta_{\lambda}(K)$ for every $K \subseteq \mathbb{N}$.

The number sequence $x=\left(x_{j}\right)$ is said to be $\lambda$-statistically convergent to the number $\ell$ if, for each $\epsilon>0, \delta_{\lambda}\left(K_{\epsilon}\right)=0$, where $K_{\epsilon}=\left\{j \in \mathbb{N}:\left|x_{j}-\ell\right|>\epsilon\right\}$; that is,

$$
\lim _{n} \frac{1}{\lambda_{n}}\left|\left\{j \in I_{n}:\left|x_{j}-\ell\right|>\epsilon\right\}\right|=0
$$


In this case we write $S_{\lambda}-\lim _{j} x_{j}=\ell$ and we denote the set of all $\lambda$-statistically convergent sequences by $S_{\lambda}$.

A distribution function is an element of $\Delta^{+}$, where $\Delta^{+}=$ $\{f: \mathbb{R} \rightarrow[0,1] ; f$ is left-continuous, nondecreasing, $f(0)=$ 0 and $f(+\infty)=1\}$ and the subset $D^{+} \subseteq \Delta^{+}$is the set $D^{+}=\left\{f \in \Delta^{+} ; l^{-} f(+\infty)=1\right\}$. Here $l^{-} f(+\infty)$ denotes the left limit of the function $f$ at the point $x$. The space $\Delta^{+}$is partially ordered by the usual pointwise ordering of functions; that is, $f \leq g$ if and only if $f(x) \leq g(x)$ for all $x \in \mathbb{R}$.

A triangle function is a binary operation on $\Delta^{+}$, namely, a function $\tau: \Delta^{+} \times \Delta^{+} \rightarrow \Delta^{+}$that is associative, commutative nondecreasing and which has $e$ as unit; that is, for all $f, g, h \in$ $\Delta^{+}$, we have

(i) $\tau(\tau(f, g), h)=\tau(f, \tau(g, h))$,

(ii) $\tau(f, g)=\tau(g, f)$,

(iii) $\tau(f, h)=\tau(g, h)$ whenever $f \leq g$,

(iv) $\tau(f, e)=f$.

Here $e$ is the d.f. defined by

$$
e(x)= \begin{cases}0 & \text { if } x \leq 0 \\ 1 & \text { if } x>0\end{cases}
$$

We remark that the set $\Delta$ as well as its subsets can be partially ordered by the usual pointwise order: in this order, $e$ is the maximal element in $\Delta^{+}$.

There are two definitions of probabilistic normed space, the original one by Šerstnev [19] who used the idea of Menger [20] to define such space and the other one by Alsina et al. [21].

According to Šerstnev, a probabilistic normed space (for short, $\mathrm{PN}$-space) is a triple $(X, v, \tau)$, where $X$ is a real linear space, $\tau$ is a triangle function, and $\nu$ is the probabilistic norm; that is, $v$ is a map from $X$ into $\Delta^{+}$that satisfies the following conditions:

(i) $\nu_{x}=e$ if and only if $x=\theta$, where $\theta$ is the null vector of $X$,

(ii) $\nu_{\alpha x}(t)=v_{x}(t /|\alpha|)$ for all $t>0, \alpha \in \mathbb{R}$ with $\alpha \neq 0$, and $x \in X$,

(iii) $v_{x+y} \geq \tau\left(v_{x}, v_{y}\right)$ whenever $x, y \in X$.

Here $v_{x}(t)$ denotes the value of $v_{x}$ at $t \in \mathbb{R}$.

In this paper, using the notions of statistical convergence and de la Vallée-Poussin mean, we define and study a new type of summability methods in the setting of probabilistic normed spaces. We also introduce a new type of statistical completeness through de la Vallée-Poussin mean in this framework.

\section{Statistical Summability through de la Vallée-Poussin Mean}

Here we introduce the notions of $\lambda$-summable and statistically $\lambda$-summable in $\mathrm{PN}$-space and give some of its properties. We will assume throughout this paper that $(X, \mathscr{F}, \tau)$ is a probabilistic normed space.
Definition 1. A sequence $x=\left(x_{k}\right)$ is said to be $\lambda$-summable in PN-space $(X, \nu, \tau)$ or simply $(\lambda)_{\nu}$-summable to $\xi$ if for each $\varepsilon>0, \theta \in(0,1)$ there exists a positive integer $j_{0}$ such that $\nu_{t_{j}(x)-\xi}(\varepsilon)>1-\theta$ for all $j \geq j_{0}$. In this case one writes $\nu(\lambda)$ - $\lim x_{k}=\xi$ and $\xi$ is called the $\nu(\lambda)$-limit of the sequence $x=\left(x_{k}\right)$.

Definition 2. A sequence $x=\left(x_{k}\right)$ is said to be statistically $\lambda$-summable in $(X, \nu, \tau)$ or simply $S(\lambda)$-summable to $\xi$ if for each $\varepsilon>0, \theta \in(0,1)$ the set $K_{\varepsilon}(\lambda)=\left\{j \in \mathbb{N}: v_{t_{j}(x)-\xi}(\varepsilon) \leq\right.$ $1-\theta\}$ has natural density zero (briefly, $\delta\left(K_{\varepsilon}(\lambda)\right)=0$ ); that is,

$$
\lim _{n} \frac{1}{n}\left|\left\{j \leq n: v_{t_{j}(x)-\xi}(\varepsilon) \leq 1-\theta\right\}\right|=0 .
$$

In this case one writes $\nu\left(S_{\lambda}\right) \lim x_{k}=\xi$, and $\xi$ is called the $\nu\left(S_{\lambda}\right)$-limit of $x$. One may write $(8)$ in the alternative form as

$$
\lim _{n} \frac{1}{n}\left|\left\{j \leq n: v_{t_{j}(x)-\xi}(\varepsilon)>1-\theta\right\}\right|=1 .
$$

Theorem 3. If a sequence $x=\left(x_{k}\right)$ is statistically $\lambda$-summable in PN-space, that is, $v\left(S_{\lambda}\right)-\lim x_{k}=\xi$ exists, then it is unique.

Proof. Suppose that there exist two elements $\xi_{1}, \xi_{2} \in X$ with $\xi_{1} \neq \xi_{2}$ such that $\nu\left(S_{\lambda}\right)-\lim x_{k}=\xi_{1}$ and $\nu\left(S_{\lambda}\right)-\lim x_{k}=\xi_{2}$. Let $\epsilon>0$ be given. Choose $q>0$ such that

$$
\tau((1-q),(1-q))>1-\epsilon .
$$

Then, for any $t>0$, we define

$$
\begin{aligned}
& M_{q}^{\prime}(\lambda)=\left\{j \in \mathbb{N}: v_{t_{j}(x)-\xi_{1}}(t) \leq 1-q\right\}, \\
& M_{q}^{\prime \prime}(\lambda)=\left\{j \in \mathbb{N}: v_{t_{j}(x)-\xi_{2}}(t) \leq 1-q\right\} .
\end{aligned}
$$

Since $\nu\left(S_{\lambda}\right)-\lim x=\xi_{1}$ implies $\delta\left(M_{q}^{\prime}(\lambda)\right)=0$ and, similarly, we have $\delta\left(M_{q}^{\prime \prime}(\lambda)\right)=0$. Now, let $M_{q}(\lambda)=M_{q}^{\prime}(\lambda) \cap M_{q}^{\prime \prime}(\lambda)$. It follows that $\delta\left(M_{q}(\lambda)\right)=0$ and hence the complement $M_{q}^{c}(\lambda)$ is nonempty set and $\delta\left(M_{q}^{c}(\lambda)\right)=1$. Now, if $k \in \mathbb{N} \backslash M_{q}(\lambda)$, then

$$
\begin{aligned}
\nu_{\xi_{1}-\xi_{2}}(t) & \geq \tau\left(v_{t_{j}(x)-\xi_{1}}\left(\frac{t}{2}\right), v_{t_{j}(x)-\xi_{2}}\left(\frac{t}{2}\right)\right) \\
& >\tau((1-q),(1-q))>1-\epsilon .
\end{aligned}
$$

Since $\epsilon>0$ was arbitrary, we get $\nu_{\xi_{1}-\xi_{2}}(t)=1$ for all $t>0$. Hence $\xi_{1}=\xi_{2}$. This means that $\nu\left(S_{\lambda}\right)$-limit is unique.

The following theorem gives the algebraic properties of statistically $\lambda$-summable sequences in $\mathrm{PN}$-spaces.

Theorem 4. Let $x=\left(x_{k}\right)$ and $y=\left(y_{k}\right)$ be two sequences. If $\nu\left(S_{\lambda}\right)-\lim x_{k}=\xi_{1}$ and $\nu\left(S_{\lambda}\right)$-lim $y_{k}=\xi_{2}$, then

(i) $v\left(S_{\lambda}\right)-\lim \left(x_{k} \pm y_{k}\right)=\xi_{1} \pm \xi_{2}$,

(ii) $v\left(S_{\lambda}\right)-\lim \alpha x_{k}=\alpha \xi, \alpha(\neq 0) \in \mathbb{R}$.

Proof of the theorem is straightforward and so omitted. 
Theorem 5. If a sequence $x=\left(x_{k}\right)$ is $\lambda$-summable to $\xi$ in $P N$ space; then it is statistically $\lambda$-summable to the same limit.

Proof. Let $\nu(\lambda)-\lim x_{k}=\xi$. Then for every $\epsilon>0$ and $t>0$, there is a positive integer $j_{0}$ such that

$$
v_{t_{j}(x)-\xi}(t)>1-\epsilon
$$

for all $j \geq j_{0}$. Since the set

$$
K_{\epsilon}(\lambda):=\left\{j \in \mathbb{N}: v_{t_{j}(x)-\xi}(t) \leq 1-\epsilon\right\}
$$

is contained in $\left\{1,2,3, \ldots, j_{0}-1\right\}$. As we know, every finite subset of $\mathbb{N}$ has natural density zero; that is, $\delta\left(K_{\epsilon}(\lambda)\right)=0$. Hence, a sequence $x=\left(x_{k}\right)$ is $S(\lambda)_{v}$-summable to $\xi$.

Remark 6. The converse of the above theorem is not true in general, which is verified by the following example.

Example 7. Let $(\mathbb{R},|\cdot|)$ denote the space of all real numbers with the usual norm and $\tau(a, b)=a b$ for all $a, b \in[0,1]$. Let $v_{x}(t)=t /(t+|x|)$ for all $x \in X$ and $t>0$. In this case, we observe that $(\mathbb{R}, v, \tau)$ is a $\mathrm{PN}$-space. Define a sequence $x=$ $\left(x_{k}\right)$ by

$$
t_{j}(x)= \begin{cases}j ; & \text { if } j=n^{2}, n \in \mathbb{N} \\ 0 ; & \text { otherwise. }\end{cases}
$$

For $\epsilon>0$ and $t>0$, write

$$
K_{\epsilon}(\lambda)=\left\{j \in \mathbb{N}: v_{t_{j}(x)}(t) \leq 1-\epsilon\right\} .
$$

It is easy to see that

$$
v_{t_{j}(x)}(t)=\frac{t}{t+\left|t_{j}(x)\right|}= \begin{cases}\frac{t}{t+j}, & \text { for } j=n^{2}, n \in \mathbb{N} ; \\ 1, & \text { otherwise; }\end{cases}
$$

hence

$$
\lim v_{t_{j}(x)}(t)= \begin{cases}0, & \text { for if } j=n^{2}, n \in \mathbb{N} \\ 1, & \text { otherwise. }\end{cases}
$$

Therefore, the sequence $\left(x_{k}\right)$ is not $(\lambda)_{\nu}$-summable. But the set $K_{\epsilon}(\lambda)$ has natural density zero since $K_{\epsilon}(\lambda) \subset\{1,4,9,16, \ldots\}$. From here, we conclude that the converse of Theorem 5 need not be true.

Theorem 8. A sequence $x=\left(x_{k}\right)$ is statistically $\lambda$-summable in $P N$-space to $\xi$ if and only if there exists a subset $K=\left\{k_{1}<k_{2}<\right.$ $\left.\cdots<k_{n}<\cdots\right\} \subseteq \mathbb{N}$ such that $\delta(K)=1$ and $\nu(\lambda)-\lim x_{k_{n}}=\xi$.

Proof. Suppose that there exists a subset $K=\left\{k_{1}<k_{2}<\right.$ $\left.\cdots<k_{n}<\cdots\right\} \subseteq \mathbb{N}$ such that $\delta_{\theta}(K)=1$ and $\nu(\lambda)-\lim x_{k_{n}}=\xi$. Then there exists a positive integer $N \in \mathbb{N}$ such that for $n \geq N$

$$
v_{t_{n}(x)-\xi}(t)>1-\epsilon
$$

Put $K_{\epsilon}(\lambda)=\left\{n \in \mathbb{N}: v_{t_{n}(x)-\xi}(t) \leq 1-\epsilon\right\}$ and $K^{\prime}=$ $\left\{k_{N+1}, k_{N+2}, \ldots\right\}$. Then $\delta\left(K^{\prime}\right)=1$ and $K_{\epsilon}(\lambda) \subseteq \mathbb{N}-K^{\prime}$ which implies that $\delta\left(K_{\epsilon}(\lambda)\right)=0$. Hence $x=\left(x_{k}\right)$ is statistically $\lambda$ summable to $\xi$ in $\mathrm{PN}$-space.

Conversely, let sequence $x=\left(x_{k}\right)$ is statistically $\lambda$ summable to $\xi$. For $q=1,2,3, \ldots$ and $t>0$, write

$$
\begin{aligned}
& K_{q}(\lambda)=\left\{j \in \mathbb{N}: v_{t_{k_{j}}(x)-\xi}(t) \geq 1-\frac{1}{q}\right\}, \\
& M_{q}(\lambda)=\left\{j \in \mathbb{N}: v_{t_{k_{j}}(x)-\xi}(t)<1-\frac{1}{q}\right\} .
\end{aligned}
$$

Then $\delta\left(K_{q}(\lambda)\right)=0$ and

$$
\begin{gathered}
M_{1}(\lambda) \supset M_{2}(\lambda) \supset \cdots M_{i}(\lambda) \supset M_{i+1}(\lambda) \supset \cdots \\
\delta\left(M_{q}(\lambda)\right)=1, \quad q=1,2, \ldots
\end{gathered}
$$

Now we have to show that for $j \in M_{q}(\lambda), x=\left(x_{k_{j}}\right)$ is $(\lambda)_{v}$-summable to $\xi$. Suppose that $x=\left(x_{k_{j}}\right)$ is not $(\lambda)_{v^{-}}$ summable to $\xi$. Therefore there is $\epsilon>0$ such that $\nu_{t_{k_{j}}-\xi}(t) \geq \epsilon$ for infinitely many terms. Let

$$
M_{\epsilon}(\lambda)=\left\{j \in \mathbb{N}: v_{t_{k_{j}}-\xi}(t)<\epsilon\right\},
$$

and $\epsilon>1 / q$ with $q=1,2,3 \ldots$ Then

$$
\delta\left(M_{\epsilon}(\lambda)\right)=0
$$

and, by (21), $M_{q}(\lambda) \subset M_{\epsilon}(\lambda)$. Hence $\delta\left(M_{q}(\lambda)\right)=0$, which contradicts $(22)$ and therefore $x=\left(x_{k_{j}}\right)$ is $(\lambda)_{v}$-summable to $\xi$.

Similarly we can prove the following dual statement.

Theorem 9. A sequence $x=\left(x_{k}\right)$ is $\lambda$-statistically summable in $P N$-space to $\xi$ if and only if there exists a subset $K=\left\{k_{1}<k_{2}<\right.$ $\left.\cdots<k_{n}<\cdots\right\} \subseteq \mathbb{N}$ such that $\delta_{\lambda}(K)=1$ and $\nu$ - $\lim x_{k_{n}}=\xi$.

\section{Statistically Complete through de la Vallée-Poussin Mean}

In this section, we define the notions of statistically $\lambda$-Cauchy and statistically $\lambda$-complete with respect to probabilistic normed space and prove related results.

Definition 10. A sequence $x=\left(x_{k}\right)$ is said to be statistically $\lambda$ Cauchy in $(X, v, \tau)$ or simply $S(\lambda)$-Cauchy if, for every $\epsilon>0$ and $\theta \in(0,1)$, there exists a number $N=N(\epsilon)$ such that, for all $j, h \geq N$, the set $S_{\epsilon}(\lambda)=\left\{j \in \mathbb{N}: v_{t_{j}(x)-t_{h}(x)}(\epsilon) \leq 1-\theta\right\}$ has natural density zero (briefly, $\delta\left(S_{\epsilon}(\lambda)\right)=0$ ); that is,

$$
\lim _{n} \frac{1}{n}\left|\left\{j \leq n: v_{t_{j}(x)-t_{h}(x)}(\epsilon) \leq 1-\theta\right\}\right|=0 .
$$

Theorem 11. A sequence $x=\left(x_{k}\right)$ is statistically $\lambda$-summable in PN-space; then it is statistically $\lambda$-Cauchy.

Proof. Suppose that $\nu\left(S_{\lambda}\right)$-lim $x_{k}=\ell$. Let $\epsilon>0$ be a given number and choose $q>0$ such that

$$
\tau((1-q),(1-q))>1-\epsilon .
$$


Then, for $t>0$, we have $\delta\left(A_{q}(\lambda)\right)=0$, where $A_{q}(\lambda)=\{j \in$ $\left.\mathbb{N}: v_{t_{j}(x)-\ell}(t / 2) \leq 1-q\right\}$ which implies that

$$
\delta\left(A_{q}^{c}(\lambda)\right)=\delta\left(\left\{j \in \mathbb{N}: v_{t_{j}(x)-\ell}\left(\frac{t}{2}\right)>1-q\right\}\right)=1 .
$$

Let $m \in A_{q}^{c}(\lambda)$. Then $v_{t_{m}(x)-\ell}(t / 2)>1-q$.

Now, let

$$
B_{\epsilon}(\lambda)=\left\{j \in \mathbb{N}: v_{t_{j}(x)-t_{m}(x)}(t) \leq 1-\epsilon\right\} .
$$

We need to show that $B_{\epsilon}(\lambda) \subset A_{q}(\lambda)$. Let $j \in B_{\epsilon}(\lambda)$. Then $v_{t_{j}(x)-t_{m}(x)}(t) \leq 1-\epsilon$ and hence $v_{t_{j}(x)-\ell}(t / 2) \leq 1-q$; that is, $j \in A_{q}(\lambda)$. Otherwise, if $v_{t_{j}(x)-\ell}(t / 2)>1-q$, then

$$
\begin{aligned}
1-\epsilon & \geq v_{t_{j}(x)-t_{m}(x)}(t) \geq \tau\left(v_{t_{j}(x)-\ell}\left(\frac{t}{2}\right), v_{t_{m}(x)-\ell}\left(\frac{t}{2}\right)\right) \\
& >\tau((1-q),(1-q))>1-\epsilon,
\end{aligned}
$$

which is not possible. Therefore $B_{\epsilon}(\lambda) \subset A_{q}(\lambda)$ and hence a sequence $x$ is statistically $\lambda$-Cauchy in $\mathrm{PN}$-space.

Definition 12. Let $(X, v, \tau)$ be a PN-space. Then,

(i) PN-space is said to be complete if every Cauchy sequence is convergent in $(X, \nu, \tau)$;

(ii) $\mathrm{PN}$-space is said to be statistically $\lambda$-complete or simply $S(\lambda)$,-complete if every statistically $\lambda$-Cauchy sequence in $(X, \nu, \tau)$ is statistically $\lambda$-summable.

Theorem 13. Every probabilistic normed space $(X, \nu, \tau)$ is statistically $\lambda$-complete but not complete in general.

Proof. Suppose that $x=\left(x_{k}\right)$ is statistically $\lambda$-Cauchy in PNspace but not statistically $\lambda$-summable. Then there exists $M \in$ $\mathbb{N}$ such that

$$
\begin{aligned}
& \delta\left(E_{\epsilon}(\lambda)\right)=\delta\left(\left\{j \in \mathbb{N}: v_{t_{j}(x)-t_{m}(x)}(t) \leq 1-\epsilon\right\}\right)=0 \\
& \delta\left(F_{\epsilon}(\lambda)\right)=\delta\left(\left\{j \in \mathbb{N}: v_{t_{j}(x)-\ell}\left(\frac{t}{2}\right)>1-\epsilon\right\}\right)=0 .
\end{aligned}
$$

This implies that $\delta\left(F_{\epsilon}^{c}(\lambda)\right)=1$. Since

$$
v_{t_{j}(x)-t_{m}(x)}(t) \geq 2 v_{t_{j}(x)-\ell}\left(\frac{t}{2}\right)>1-\epsilon,
$$

if $v_{t_{j}(x)-\ell}(t / 2)>(1-\epsilon) / 2$, then $\delta\left(E_{\epsilon}^{c}(\lambda)\right)=0$; that is, $\delta\left(E_{\epsilon}(\lambda)\right)=1$, which leads to a contradiction, since $x=$ $\left(x_{k}\right)$ was statistically $\lambda$-Cauchy. Hence $x=\left(x_{k}\right)$ must be statistically $\lambda$-summable in $\mathrm{PN}$-space.

A probabilistic normed space is not complete in general; we verify this by the following example.

Example 14. Let $X=(0,1)$ and $v_{x}(t)=t /(t+|x|)$ for $t>0$. Then $(X, \nu, \tau)$ is a probabilistic normed space but not complete, since the sequence $(1 /(n+1))$ is Cauchy with respect to $(X, v, \tau)$ but not convergent with respect to the present $\mathrm{PN}$-space.

\section{Conflict of Interests}

The author declares that there is no conflict of interests regarding the publication of this paper.

\section{Acknowledgment}

The author thanks the referees for their comments.

\section{References}

[1] H. Fast, "Sur la convergence statistique," Colloquium Mathematicum, vol. 2, pp. 241-244, 1951.

[2] J. A. Fridy, “On statistical convergence," Analysis, vol. 5, no. 4, pp. 301-313, 1985.

[3] M. Mursaleen and S. A. Mohiuddine, "On ideal convergence in probabilistic normed spaces," Mathematica Slovaca, vol. 62, no. 1, pp. 49-62, 2012.

[4] M. Mursaleen and A. Alotaibi, "Statistical summability and approximation by de la Vallée-Poussin mean," Applied Mathematics Letters, vol. 24, no. 3, pp. 320-324, 2011.

[5] B. C. Tripathy, "On genralized difference paranormed statistically convergent sequences," Indian journal of Pure and Applied Mathematics, vol. 35, no. 5, pp. 655-663, 2004.

[6] B. C. Tripathy and H. Dutta, "On some lacunary difference sequence spaces defined by a sequence of Orlicz functions and $\Delta_{m}^{n}$-statistical convergence," Analele Ştiinţifice ale Universităţii Ovidius, Seria Matematica, vol. 20, no. 1, pp. 417-430, 2012.

[7] B. C. Tripathy, A. Baruah, M. Et, and M. Gungor, "On almost statistical convergence of new type of generalized difference sequence of fuzzy numbers," Iranian Journal of Science and Technology, Transactions A: Science, vol. 36, no. 2, pp. 147-155, 2012.

[8] B. C. Tripathy and A. Baruah, "Lacunary statically convergent and lacunary strongly convergent generalized difference sequences of fuzzy real numbers," Kyungpook Mathematical Journal, vol. 50, no. 4, pp. 565-574, 2010.

[9] Mursaleen, " $\lambda$-statistical convergence," Mathematica Slovaca, vol. 50, no. 1, pp. 111-115, 2000.

[10] R. Çolak and Ç. A. Bektas, " $\lambda$-statistical convergence of order $\alpha$," Acta Mathematica Scientia B, vol. 31, no. 3, pp. 953-959, 2011.

[11] S. Karakus, "Statistical convergence on probabilistic normed space," Mathematical Communications, vol. 12, pp. 11-23, 2007.

[12] H. Çakalli, "Lacunary statistical convergence in topological groups," Indian Journal of Pure and Applied Mathematics, vol. 26, no. 2, pp. 113-119, 1995.

[13] H. Çakalli and M. K. Khan, "Summability in topological spaces," Applied Mathematics Letters, vol. 24, no. 3, pp. 348-352, 2011.

[14] S. A. Mohiuddine and M. Aiyub, "Lacunary statistical convergence in random 2-normed spaces," Applied Mathematics \& Information Sciences, vol. 6, no. 3, pp. 581-585, 2012.

[15] S. A. Mohiuddine, A. Alotaibi, and M. Mursaleen, "Statistical convergence of double sequences in locally solid Riesz spaces," Abstract and Applied Analysis, vol. 2012, Article ID 719729, 9 pages, 2012.

[16] M. Mursaleen, V. Karakaya, M. Ertürk, and F. Gürsoy, "Weighted statistical convergence and its application to Korovkin type approximation theorem," Applied Mathematics and Computation, vol. 218, no. 18, pp. 9132-9137, 2012. 
[17] E. Savas and S. A. Mohiuddine, " $\bar{\lambda}$-statistically convergent double sequences in probabilistic normed spaces," Mathematica Slovaca, vol. 62, no. 1, pp. 99-108, 2012.

[18] B. C. Tripathy, M. Sen, and S. Nath, "I-convergence in probabilistic n-normed space," Soft Computing, vol. 16, no. 6, pp. 10211027, 2012.

[19] A. N. Šerstnev, "Random normed spaces: problems of completeness," Kazanskii Gosudarstvennyi Universitet Uchenye Zapiski, vol. 122, no. 4, pp. 3-20, 1962.

[20] K. Menger, "Statistical metrics," Proceedings of the National Academy of Sciences of the United States of America, vol. 28, no. 12, pp. 535-537, 1942.

[21] C. Alsina, B. Schweizer, and A. Sklar, "On the definition of a probabilistic normed space," Aequationes Mathematicae, vol. 46, no. 1-2, pp. 91-98, 1993. 


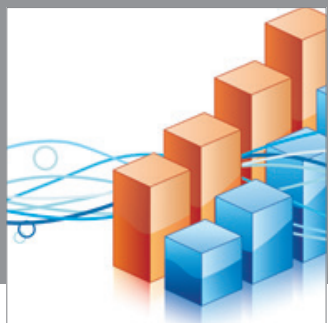

Advances in

Operations Research

mansans

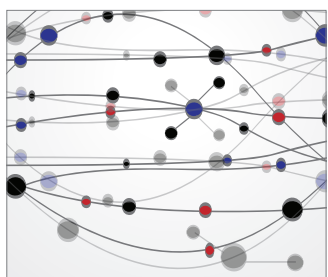

The Scientific World Journal
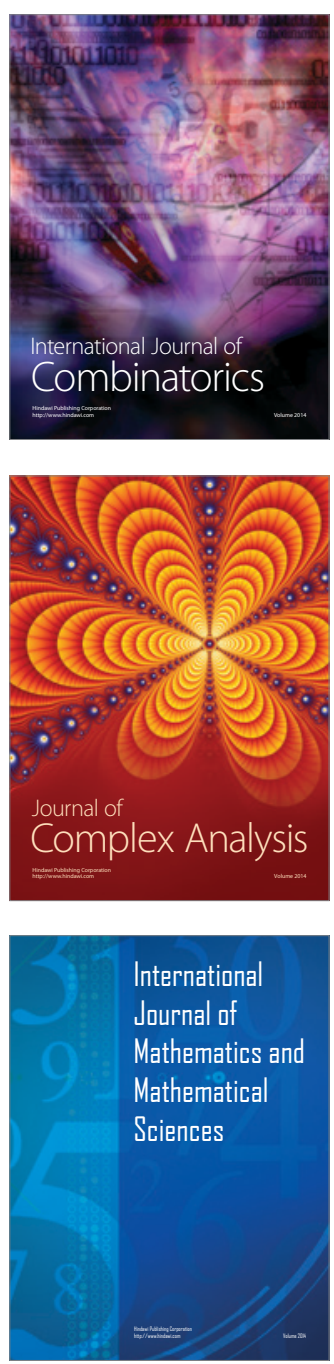
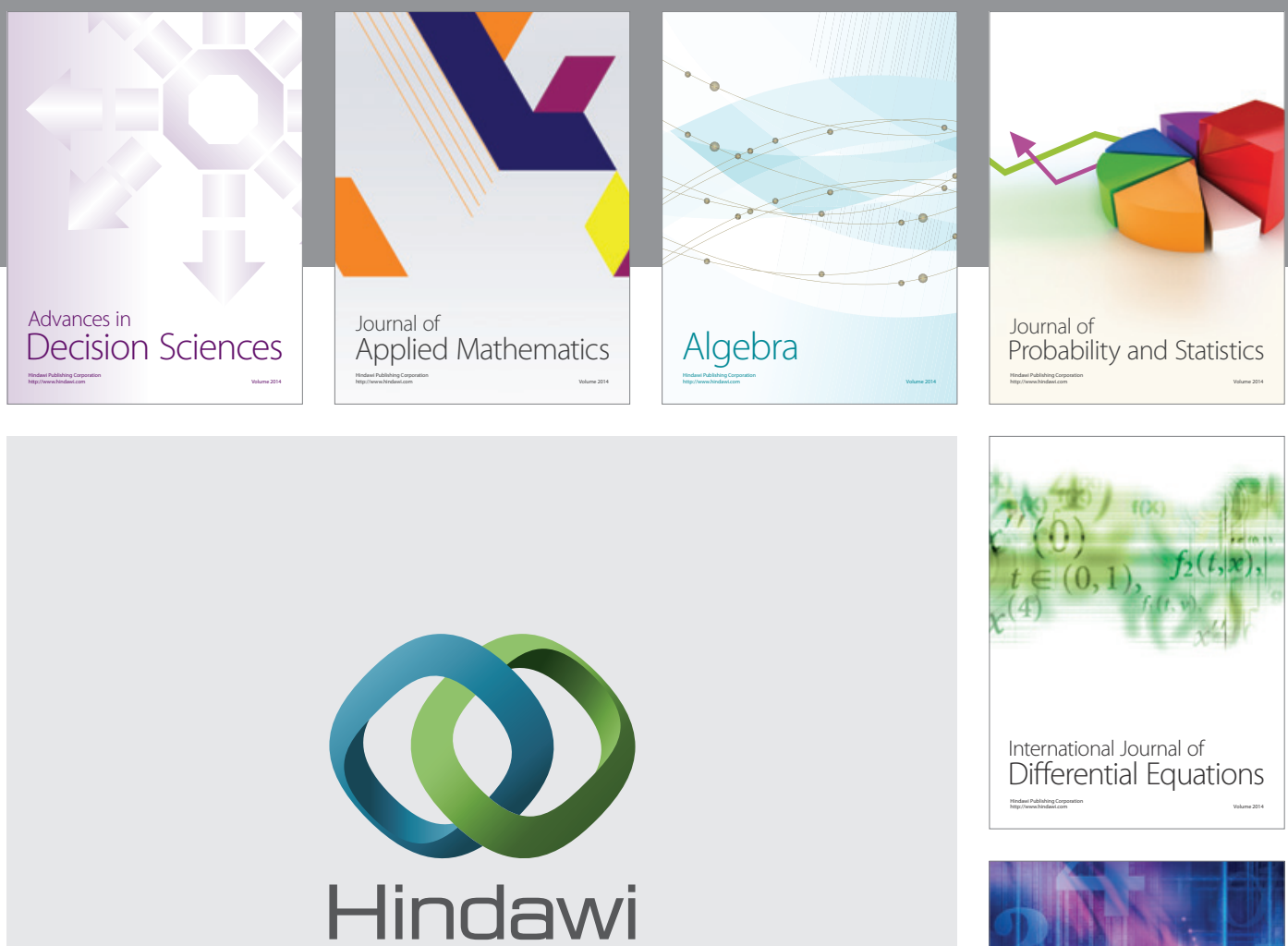

Submit your manuscripts at http://www.hindawi.com
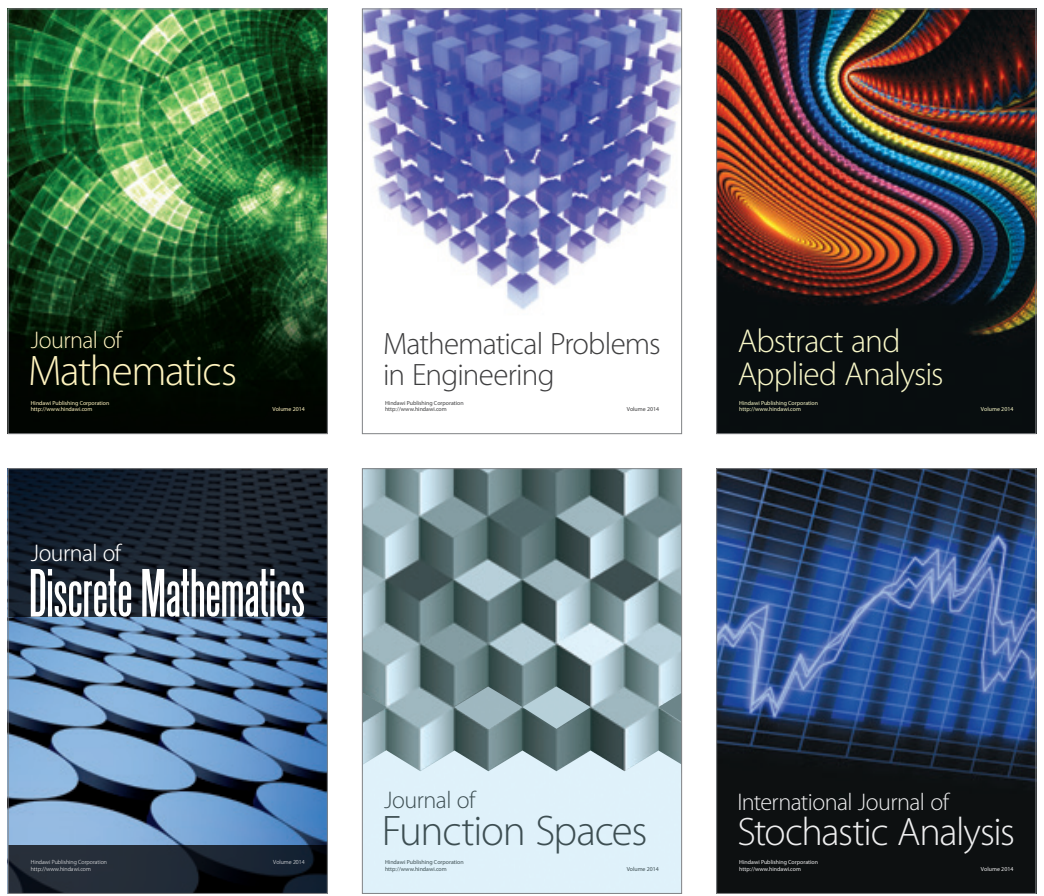

Journal of

Function Spaces

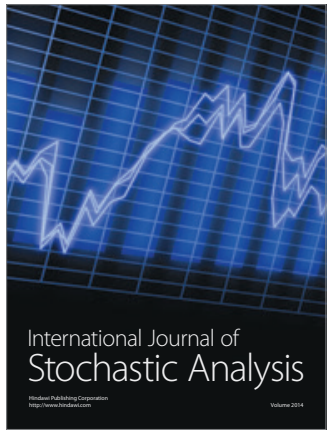

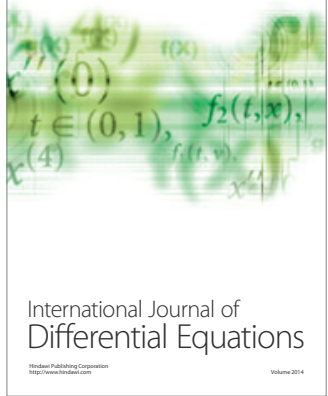
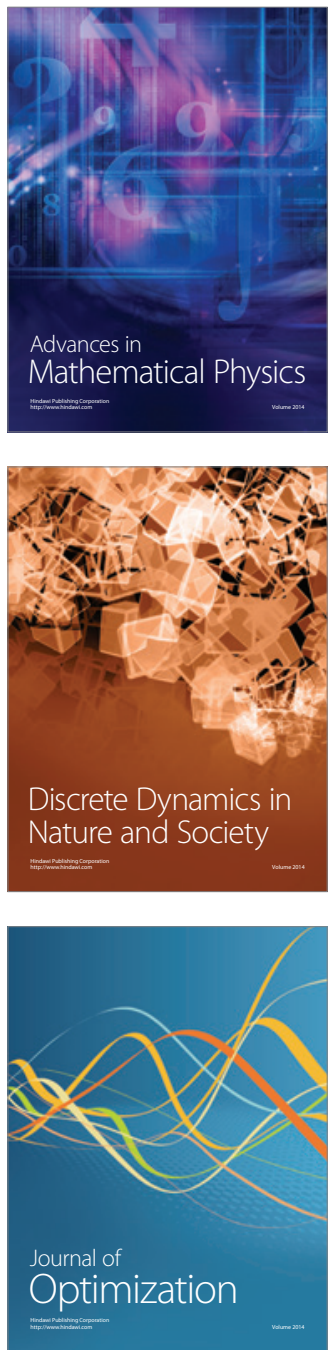